ORIGINAL

\title{
Estudio de razas de palomas españolas a partir del análisis de caracteres morfológicos cualitativos
}

\author{
Study of spanish dove breeds based on the analysis of qualitative \\ morphological characteristics
}

Pere-Miquel Parés C, Ph. D.

Universidad de Lleida, ETSEA, Departamento de Producción Animal. Avda. Rovira Roure 191, 25198-Lleida, Catalunya, España. Correspondencia: peremiquelp@prodan.udl.cat

Recibido: Febrero 16 de 2010; Aceptado: Agosto 15 de 2010

\section{RESUMEN}

Objetivo. Evaluar una posible base morfológica que sustente una clasificación racional y no arbitraria de razas de palomo, se estudió la relación fenética entre diferentes razas. Materiales y métodos. Se sometieron para su estudio morfológico comparativo un total de 29 razas españolas de palomas, considerándose cada raza como Operational Taxonomic Unit (OTU) y agrupadas a priori en grupos de afinidad funcional y morfológica: buchones, razas catalanas, razas baleáricas, palomos de morfología. Se realizó un análisis de coordenadas (ACo) sobre la base de la matriz de distancias de similitud de Gower entre OTUs a partir del análisis de 29 caracteres. A fin de conocer cuáles eran las variables responsables de las similitudes y/o disimilitudes observadas se aplicó igualmente un análisis de correspondencia (ACrr). Resultados. En el ACo se reflejó que la variabilidad representada por los dos primeros vectores Eigen no es alta. El ACrr demostró que muchas variables están fuertemente correlacionadas. Conclusiones. La distribución de las razas en el ACo apunta a una distribución bastante buena en los 4 grupos establecidos. El ACrr mostró que las variables más discriminantes pueden ser reducidas a nueve: grosor y longitud del pico, desarrollo de las carúnculas nasales y oculares, longitud del cuello, tamaño y colgado de buche, longitud relativa de la cola y posición de las alas en reposo respecto de la cola.

Palabras clave: Análisis de coordenadas, análisis de correspondencia, buchón ladrón, etnología, morfología, variedad.

\section{ABSTRACT}

Objective. To evaluate a possible morphological base that supports a rational and nonarbitrary classification of dove breeds, the phenetic relationship between different breeds was studied. Materials and methods. Twenty nine different dove breeds were submitted 
for a comparative morphological study, considering each breed as an Operational Taxonomic Unit (OUT), and grouped a priori in morphological and functional affinity groups such as: large crop, Catalonian breeds, Balearic breeds, and morphological doves. A coordinate analysis (ACO) was made based on Gower's similar distance matrix between OTUs from the analysis of 29 traits. In order to know which were the variables responsible for the observed similarities or differences, a correspondence (Carr) analysis was carried out. Results. The ACo showed that the variability represented by the two first eigen vectors is not high. The Carr showed that many of the variables are strongly related. Conclusions. The distribution of breeds in the Aco points towards a fairly good distribution in the 4 established groups. The Carr demonstrated that the most discriminating variables may be reduced to nine: thickness and beak length, development of nasal and eye caruncles, neck length, crop size and hang, relative tail length, and wing position in relation to the tail at rest position.

Key words: Coordenate analysis, correspondence analysis, crop, ethnology, morphology, variety.

\section{INTRODUCCIÓN}

El pensamiento humano, en general, como se refleja en el lenguaje, parece depender fuertemente del reconocimiento de grupos, particularmente los grupos de objetos a los que damos nombres colectivos (1). Podríamos notar que "raza de paloma" implica dos procesos mentales, primero el de considerar las semejanzas que poseen "todas" las palomas y segundo, considerar las diferencias entre razas. En el contexto etnológico, el concepto de clasificación racial está restringido al agrupamiento de los animales en razas por sus atributos estructurales, excluyendo de ellas algunos animales considerados "no puros".

Muchas veces resulta empírico clasificar las razas en grupos, en base a una coincidencia de caracteres, y por ello la relación que suele utilizarse para hacer un agrupamiento racial suele ser la "asociación por semejanza". Una insuficiencia de caracteres informativos puede ser la razón de por qué no se puede resolver la falta de una clasificación consistente. Dos fenómenos son los que podrían contribuir a esta dificultad. Primero, el hecho, bien conocido, de que el fenotipo, en la mayoría de las razas, está "estandarizado", responde a un prototipo ideal -lo que debe ser- no a un morfotipo -lo que es-. Pero el estándar proporciona solamente unos pocos indicios morfológicos, insuficientes para intentar esta asociación. El tema se complica aún más en las razas que no poseen estándar racial.
En taxonomía numérica existe un gran número de métodos de análisis multivariado tendientes a la caracterización de individuos o conjunto de individuos, generalmente asociados al tipo de variables con que se trabaja. El Análisis de Coordenadas (ACo) permite analizar las similitudes entre los perfiles de una misma nube de puntos, pudiendo establecerse qué grupos son más similares o disimilares entre sí, así como las similitudes entre categorías. Pero el ACo únicamente muestra la similitud o disimilitud entre grupos, facilitando la separación de éstos, aunque no permite saber cuáles son las variables responsables de estas similitudes y/o disimilitudes observadas. La interpretación de las relaciones entre perfiles de nubes diferentes no es directa, siendo necesario considerar la naturaleza baricéntrica de tal relación; y para ello, tales asociaciones deben evaluarse mediante las proyecciones sobre ejes factoriales. El análisis de correspondencia (ACrr) permite resaltar las variables que determinan la configuración de los ejes y que gozan de mayor calidad de representación.

En este trabajo se presenta un estudio de las relaciones existentes entre diferentes razas de palomas españolas, realizado a partir del análisis de la información generada del estudio de sus caracteres morfológicos cualitativos, con el objetivo final de convertir los resultados en un esquema de clasificación racional y, por tanto, no arbitrario. La 
ausencia hasta el presente de datos suficientemente objetivos de las razas de palomas, indujo el planteamiento de esta investigación, en un intento de contribuir a ampliar su conocimiento. Debe destacarse que los resultados se obtienen en base a semejanzas morfológicas, y no se pretende de ningún modo establecer una clasificación de tipo filogenético.

\section{MATERIALES Y MÉTODOS}

Se sometieron para su estudio morfológico comparativo un total de 29 razas de palomas, considerándose cada raza como OTU ("Operational Taxonomic Unit"), agrupadas a priori en grupos de afinidad funcional y morfológica: buchones, razas catalanas, razas baleáricas, palomos de morfología. Las razas analizadas fueron: Alteño (ALT), Borino (BBO), "Colom d'Escampadissa" (BCE), Buchón Balear o "Gavatxut" (BGA), Mallorquina (BMA), "Nas de Xot"
(BNX), "Pinta Balear" o "d'Esbart" (BPB), Canario (CAN), Colillano (COL), Gaditano (GAD), Granadino (GRA), Jiennense (JIE), Laudino Sevillano (LAU), Marteño (MAT), Marchenero $(\mathrm{MCH})$, Moroncelo (MOR), Morrillero Alicantino (MRR), Quebrado Murciano (QUE), Rafeño (RAF), "Coll Pelat" (SCP), "Colom d'UII" (SCU), Figureta (SFI), "Flamenquilla" (SFL), Refilador o "Ull de Peix" (SRE), "Ull de Maduixa" (SUM), "Vol Català" (SVC), Chorrera o "Colom d'Enreixat" (SXO), Veleño (VEL) y Nuevo Buchón Valenciano (NVP). Como grupo de comparación se utilizó la paloma bravía (Columba livia, PAB).

Se definieron 29 caracteres morfológicos, todos ellos morfológicos cualitativos discontinuos y cualitativos, en series desordenadas (no aditivas y, lógicamente, no de naturaleza lineal), y dicotómicas o, la mayoría, multicotómicas. El estado de cada carácter para cada raza se estableció a partir de las descripciones dadas por el patrón

Tabla 1. Caracteres utilizados y su correspondiente codificación

\begin{tabular}{|c|c|}
\hline (A) Peso & 1. Elipométrico, 2. Eumétrico, 3. Subhipermétrico, 4. Hipermétrico \\
\hline (B) Tamaño & 1. Muy pequeño, 2. Pequeño, 3. Mediano, 4. Grande, 5. Muy grande \\
\hline (C) Tamaño relativo de la cabeza & 1. Pequeño, 2. Mediano, 3. Grande \\
\hline (D) Anchura de la cabeza & 1. Estrecha, 2. Mediana, 3. Ancha \\
\hline (E) Forma de la cabeza & 1. Redondeada, 2. Cuadrada, 3. Alargada ("en almendra"), 4. Muy redondeada ("acarnerada") \\
\hline (F) Grosor del pico & 1. Fino, 2. Medio, 3. Grueso \\
\hline (G) Longitud del pico & 1. Muy corto, 2. Corto, 3. Medio, 4. Largo \\
\hline (H) Forma del pico & 1. Rectilíneo, 2. Ligeramente curvado, 3. Curvado, 4. Muy curvado ("de mochuelo") \\
\hline (I) Desarrollo de las carúnculas nasales & 1. Escasas, 2. Ligeramente desarrolladas, 3. Desarrolladas, 4. Muy desarrolladas \\
\hline (J) Aspecto de las carúnculas nasales & 1. Finas, 2. Rugosas \\
\hline (K) Desarrollo de las carúnculas oculares & 1. Finas, 2. Desarrolladas, 3. Muy desarrolladas \\
\hline (L) Cresta & 1. Ausente, 2. Ausente o presente \\
\hline (M) Longitud del cuello & 1. Corto, 2. Medio, 3. Largo \\
\hline (N) Grosor del cuello & 1. Delgado, 2. Medio, 3. Grueso \\
\hline (0) Engrifado en el cuello ("morrillo") & 1. Si, 2. No \\
\hline (P) Garlanda & 1. Si, 2. No \\
\hline (Q) Anchura de pecho & 1. Medio, 2. Ancho, 3. Muy ancho \\
\hline (R) Longitud de piernas & 1. Cortas, 2. Medias, 3. Largas \\
\hline (S) Grosor de piernas & 1. Finas, 2. Medias, 3. Gruesas \\
\hline (T) Tamaño del buche & 1. Pequeño, 2. Mediano, 3. Bien desarrollado \\
\hline (U) Colgado de buche & 1. No colgante, 2. Ligeramente colgado, 3. Colgado, 4. Péndulo \\
\hline (V) Rafe & 1. Si, 2. No, 3. Si o no \\
\hline (W) Perfil del dorso & 1. Llano, 2. Ligeramente arqueado, 3. Arqueado \\
\hline (X) Anchura del obispillo & 1. Medio, 2. Ancho, 3. Muy ancho \\
\hline (Y) Longitud relativa de la cola & 1. Cortas, 2. Medias, 3. Largas \\
\hline (Z) Forma de la cola & 1. Arqueada, 2. Llana \\
\hline (AA) Postura corporal en reposo & 1. Erecta, 2. Horizontal \\
\hline (AB) Alcance punta de las alas-cola en reposo & 1. Alcanzan la punta de la cola, 2. No alcanzan la punta de la cola, 3. Superan la punta de la cola \\
\hline (AC) Posición de las alas en reposo respecto de la cola & 1. Encima de la cola, 2. Debajo de la cola, 3. Al mismo nivel que la cola, 4. Encima o debajo de la cola \\
\hline
\end{tabular}


racial (en las razas que lo poseen) y de las descripciones proporcionadas por Levy (2), Mackrott (3) y Schille (4) y, en caso de falta de algún dato en la bibliografía consultada, según las informaciones ofrecidas por los propios criadores. Los números para cada estado fueron asignados de una manera arbitraria, no implicando ningún peso específico. El número de estado para cada carácter fue establecido según el número de clases fenotípicas distinguibles. Cada estado puede derivarse directamente de cualquier otro y en cualquier secuencia, por lo que los caracteres son tratados bajo la opción de no ser aditivos (1). Se contabiliza como máximo un paso por transformación al aplicarlo a la longitud del árbol (1). Los caracteres utilizados y su correspondiente codificación se muestran en la tabla 1 . La matriz básica de datos (MBD) se presenta en la tabla 2.

A fin de separar OTUs con características similares, se realizó un análisis de coordenadas (ACo) sobre la base de la matriz de distancias de similitud de Gower entre OTUs, aplicando una transformación exponencial $c=1$. El coeficiente de similitud de Gower (5) permite que los valores evaluados involucren variables de diferente naturaleza (nominal, presencia/ausencia), siendo apto para calcular similitudes cuando se tiene tales mezclas de variables, como es el caso del presente estudio.

Tabla 2. Matriz básica de datos (MBD). Abreviaturas en el texto

\begin{tabular}{|c|c|c|c|c|c|c|c|c|c|c|c|c|c|c|c|c|c|c|c|c|c|c|c|c|c|c|c|c|c|}
\hline & $F$ & & 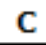 & & & & ( & H & 1 & J & 6 & L & 1 & $\mathbf{N}$ & 0 & & $\mathbf{Q}$ & $\mathbf{R}$ & 5 & $\mathbf{T}$ & $\mathbf{U}$ & V & W & & $\mathbf{Y}$ & & AA & AB & $A C$ \\
\hline 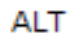 & 2 & 3 & 2 & 2 & 1 & 3 & 3 & 3 & 2 & 1 & 1 & 1 & 2 & 2 & 2 & & 1 & 1 & 3 & 3 & 4 & 2 & 3 & 1 & 2 & & 1 & 1 & 1 \\
\hline 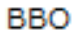 & 2 & 3 & 1 & 2 & 1 & 2 & 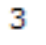 & 1 & 2 & 1 & 1 & 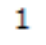 & 1 & 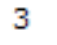 & 2 & & 2 & & 2 & 1 & 1 & & & & 3 & 2 & 2 & 1 & \\
\hline $\mathrm{CE}$ & 2 & 3 & 2 & 2 & 1 & 1 & 3 & 1 & 1 & 1 & 1 & 1 & 1 & 2 & 2 & & 1 & & 2 & 1 & 1 & & & & 3 & & 2 & 1 & 4 \\
\hline A & 2 & 3 & 2 & 2 & 1 & 3 & 2 & 2 & 2 & 1 & 1 & 1 & 1 & 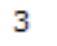 & 2 & & 3 & 1 & 1 & 2 & 2 & & & & 2 & & 1 & 2 & 1 \\
\hline . & 4 & 5 & 2 & 2 & 1 & 2 & 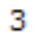 & 2 & 2 & 1 & 1 & 2 & 1 & 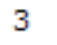 & 2 & & 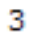 & & 2 & 1 & 1 & & & & 3 & & 2 & & 2 \\
\hline NX & 2 & 3 & 2 & 2 & 3 & 2 & 3 & 3 & 1 & 1 & 3 & 1 & 2 & 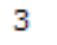 & 2 & & 1 & & 2 & 1 & L & & & & 2 & & 1 & 2 & 1 \\
\hline$B$ & 1 & 3 & 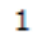 & 2 & 3 & 2 & 2 & 2 & 1 & 1 & 1 & 1 & 1 & 2 & 2 & & 2 & 2 & 2 & 1 & 1 & & & & 3 & & 2 & 2 & 2 \\
\hline AN & 2 & 3 & 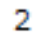 & 2 & 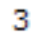 & 3 & 2 & 2 & 3 & 2 & 1 & 1 & 2 & 2 & 2 & & 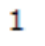 & & 2 & 3 & 4 & & & & 1 & & 2 & 1 & 1 \\
\hline L & 2 & 3 & 2 & 2 & 1 & 1 & 3 & 2 & 1 & 1 & 1 & 1 & $J$ & 3 & 2 & & 2 & 2 & 2 & 3 & J & 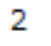 & 1 & & 2 & & 1 & 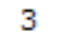 & 1 \\
\hline AD & 1 & 3 & 2 & 1 & 4 & 1 & 2 & 2 & 3 & 1 & 1 & 1 & 2 & 2 & 1 & & 2 & & 1 & 3 & 4 & & & & 1 & & 1 & & 1 \\
\hline 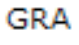 & 3 & 4 & 3 & 2 & 4 & 3 & 3 & 2 & 3 & 2 & 2 & 1 & 3 & 3 & 2 & & 2 & & 2 & 2 & & & & & 2 & & & & \\
\hline & 2 & 3 & 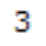 & 2 & 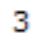 & 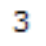 & 7 & 2 & 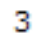 & 2 & 2 & 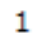 & 3 & 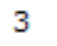 & 2 & & 2 & & 2 & 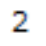 & 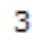 & & & & 2 & & 2 & 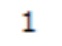 & 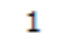 \\
\hline LAU & 2 & 3 & 2 & 2 & 3 & 3 & 3 & 2 & 4 & 1 & 1 & 1 & J & 2 & 2 & & 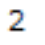 & 2 & 2 & 3 & 4 & 1 & 1 & 2 & 2 & 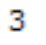 & 1 & 1 & 1 \\
\hline MAT & 3 & 3 & 2 & 2 & 1 & 1 & 2 & 3 & 2 & 1 & 1 & 1 & 3 & 2 & 2 & & 1 & & 3 & 2 & 2 & 2 & 1 & & 2 & & 1 & 1 & 1 \\
\hline 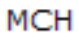 & 2 & 2 & 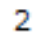 & . & - & - & 2 & 2 & 1 & 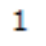 & 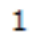 & 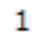 & 1 & - & 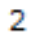 & & 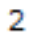 & & - & 3 & 3 & & & & 1 & & 2 & 1 & \\
\hline M & 2 & 2 & 2 & 2 & 3 & 2 & 3 & 2 & 2 & 1 & 1 & 1 & 3 & 1 & 1 & & 1 & 2 & 2 & 1 & 1 & 2 & 1 & & 2 & 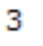 & 1 & 1 & \\
\hline MRR & 3 & 3 & 2 & 2 & 1 & 1 & 3 & 2 & 1 & 1 & 1 & 1 & 3 & 1 & 1 & & 1 & 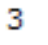 & 1 & 1 & 3 & 1 & 1 & 1 & 2 & 1 & 1 & 2 & 2 \\
\hline PAB & 2 & 3 & 1 & 1 & 1 & 1 & 2 & 1 & 1 & 1 & 1 & 1 & 1 & 2 & 2 & & 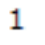 & & 2 & 1 & 1 & 2 & 1 & & 2 & & 2 & 2 & 1 \\
\hline Q & 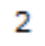 & 2 & . & 2 & . & 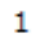 & 3 & 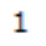 & - & 2 & 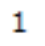 & 1 & 3 & - & 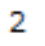 & & 2 & & 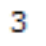 & 3 & & & & & 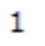 & & 2 & 1 & \\
\hline$F$ & 2 & 3 & 3 & 5 & 4 & $J$ & 1 & 4 & 4 & 1 & 2 & 1 & 1 & $n$ & 2 & & 2 & 2 & 0 & 3 & 2 & 1 & J & 2 & 1 & 2 & 1 & 1 & \\
\hline SCP & 2 & 2 & 2 & 2 & 1 & 2 & 3 & 1 & 1 & 1 & 1 & 1 & 2 & 2 & 2 & & 1 & 2 & 2 & 1 & 1 & 2 & 1 & 1 & 2 & 2 & 1 & 1 & 1 \\
\hline $\mathrm{CU}$ & 3 & 4 & 3 & 2 & 1 & 3 & 2 & 4 & 3 & 1 & 3 & 1 & 1 & 2 & 2 & & 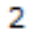 & 1 & 2 & 1 & 1 & 2 & 1 & 1 & 2 & $?$ & 2 & 1 & 1 \\
\hline SFI & 1 & 1 & + & 2 & 2 & 2 & 2 & 2 & 1 & 1 & 1 & 1 & 2 & 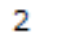 & 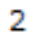 & & 2 & & 2 & - & 1 & 2 & & & 2 & & 1 & 2 & \\
\hline SFL & 2 & 2 & 3 & 2 & 2 & 3 & 2 & 2 & 3 & 1 & 3 & 1 & 1 & 2 & 2 & & 2 & 1 & 2 & . & 1 & 2 & & 1 & 2 & 2 & 1 & 1 & \\
\hline $\mathrm{E}$ & 2 & 3 & 2 & 2 & 2 & 1 & 4 & 1 & 1 & 1 & 1 & 2 & 3 & 2 & 2 & & . & & 2 & . & 1 & 2 & 1 & 1 & 2 & & 1 & 1 & 1 \\
\hline SUM & 3 & 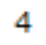 & 3 & 2 & 1 & 3 & 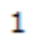 & 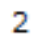 & 3 & 1 & 3 & 2 & 1 & 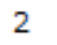 & - & & - & 1 & 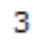 & 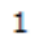 & 1 & 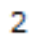 & 1 & 1 & - & $?$ & 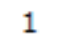 & 2 & 1 \\
\hline SVC & 2 & 2 & 1 & 2 & 1 & 1 & r & 2 & 1 & 1 & 1 & 1 & 0 & 2 & 2 & & 2 & 2 & 2 & 2 & 1 & 2 & 1 & 1 & 2 & 2 & 1 & 1 & \\
\hline SXO & 2 & 2 & 1 & 2 & 2 & 1 & 2 & 2 & 1 & 1 & 1 & 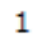 & 2 & - & 2 & & 1 & & 1 & - & 1 & 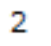 & 3 & 1 & 2 & & 1 & 1 & 1 \\
\hline$v$ & 2 & 3 & 1 & 2 & 1 & 1 & 3 & 2 & 1 & 1 & 1 & 1 & 3 & 2 & 2 & & 1 & 3 & 1 & 2 & 3 & 1 & 1 & 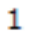 & 2 & 1 & 2 & 2 & \\
\hline VNP & 2 & 3 & - & 2 & 4 & 3 & 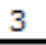 & 1 & 2 & 1 & 1 & 1 & 2 & 3 & 2 & & 2 & 2 & 3 & 2 & 2 & 3 & 1 & $?$ & 2 & 2 & 2 & 1 & \\
\hline
\end{tabular}


Quizás considerar el "peso" de los caracteres permitiría afinar esta clasificación. Y es que muchos caracteres están altamente correlacionados. Considerar pues estos caracteres correlacionados como caracteres separados podría conducir a error, puesto que hablando en términos raciales, podrían ser puramente un reflejo de un único cambio morfológico o funcional en manos de los criadores. La formación de algunas razas en base a la búsqueda de unos determinados caracteres podría conllevar, por lo menos en algunos casos, la adquisición de un considerable número de nuevos caracteres. De hecho, dentro de una raza, las transiciones entre caracteres por fuerza han de resultar más fáciles que en la evolución por mecanismos naturales ("tacañería evolutiva"). Por ello, a fin de conocer cuáles son las variables responsables de estas similitudes y/o disimilitudes observadas se aplicó un análisis de correspondencia (ACrr). Para todos los análisis se utilizó el programa PAST (6).

\section{RESULTADOS}

En el ACo se reflejó que la variabilidad representada por los dos primeros vectores Eigen no es alta. Los 3 primeros vectores absorbían únicamente un $43.37 \%$ de la variabilidad total. En la tabla 3 se muestran los eigenvalues y porcentajes para los tres primeros ejes. Se observaron los planos factoriales 1-2, 1-3 y 2-3 para evaluar en éstos la estabilidad de los cuatro grupos. Se eligió el plano 1-2 por ser el que mejor los mostraba (Figura 1) puesto que en él pueden representarse los grupos obtenidos de manera bastante exclusiva, con poca traslapación entre nubes: grupo de buchones (Alteño ALT, Canario CAN, Colillano COL, Gaditano GAD, Granadino GRA, Jiennense JIE, Laudino Sevillano LAU,

Tabla 3. Valores obtenidos del análisis de coordenadas (3 primeros ejes).

\begin{tabular}{cccc}
\hline Eje Eigenvalue Porcentaje & $\begin{array}{c}\text { Porcentaje } \\
\text { acumulado }\end{array}$ \\
\hline 1 & 0.646 & 20.95 & 20.95 \\
2 & 0.385 & 12.48 & 33.43 \\
3 & 0.307 & 9.95 & 43.38 \\
\hline
\end{tabular}

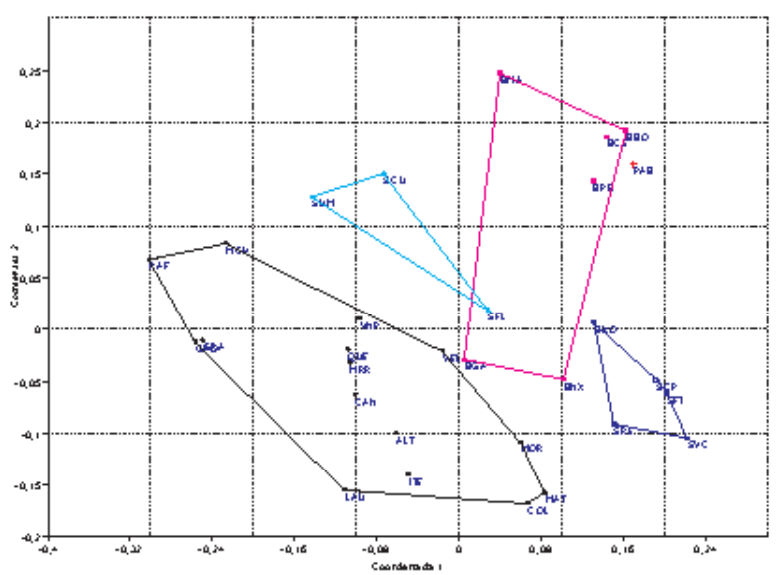

Figura 1. Análisis de Coordenadas utilizando la matriz de 29 variables cualitativas. Proyección de las OTUs en el espacio de las coordenadas 1 y 2 . Abreviaturas en el texto

Marteño MAT, Marchenero MCH, Moroncelo MOR, Morrillero Alicantino MRR, Quebrado Murciano QUE, Rafeño RAF, Veleño VEL y Nuevo Buchón Valenciano NVP), grupo 2, de palomas de vuelo funcional o acrobático ("Nas de Xot" BNX, "Coll Pelat" SCP, Figureta SFI, Refilador SRE, "Vol Català" SVC y Chorrera SXO); grupo 3, razas baleáricas (Borino BBO, "Colom d'Escampadissa" BCE, Mallorquina BMA, "Pinta Balear" BPB) de trabajo y producción (excluyendo el "Gavatxut" BGA, raza balear que entra dentro del grupo de buchones, al que pertenece funcionalmente); y grupo 4, puramente de morfología ("Colom d'UII" SCU, "Flamenquilla" SFL y "Ull de Maduixa" SUM). En el resto de los planos (figuras no mostradas) las nubes de las OTUs se distribuían uniformemente en el espacio formado por los distintos ejes.

Del ACrr (Figura 2) se desprende que muchas variables están fuertemente correlacionadas (aparecen en pequeños ángulos, como las variables $A A, A B$ y $V$, o $R$ y $W$ ), y que son 9 las de más poder discriminante: $F, G, I$, $\mathrm{K}, \mathrm{M}, \mathrm{U}, \mathrm{T}, \mathrm{Y}$ y $\mathrm{AC}$ : grosor y longitud del pico, desarrollo de las carúnculas nasales y oculares, longitud del cuello, tamaño y colgado de buche, longitud relativa de la cola y posición de las alas en reposo respecto de la cola. Un nuevo ACo con únicamente estas variables refleja un mejor ajuste de los grupos raciales (Figura 3), siendo únicamente el "Gavatxut" (BGA) el que 


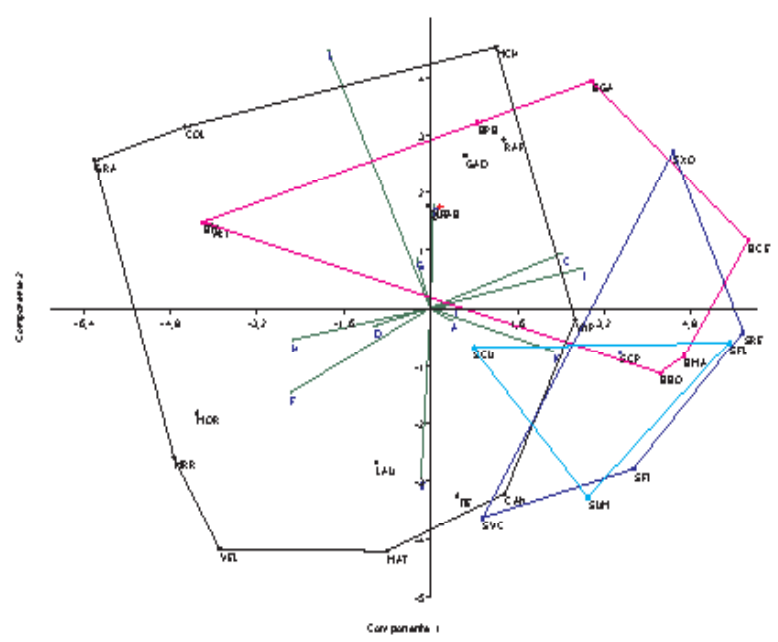

Figura 2. Análisis de Correspondencia y biplot utilizando la matriz de 29 variables cualitativas. Abreviaturas en el texto.

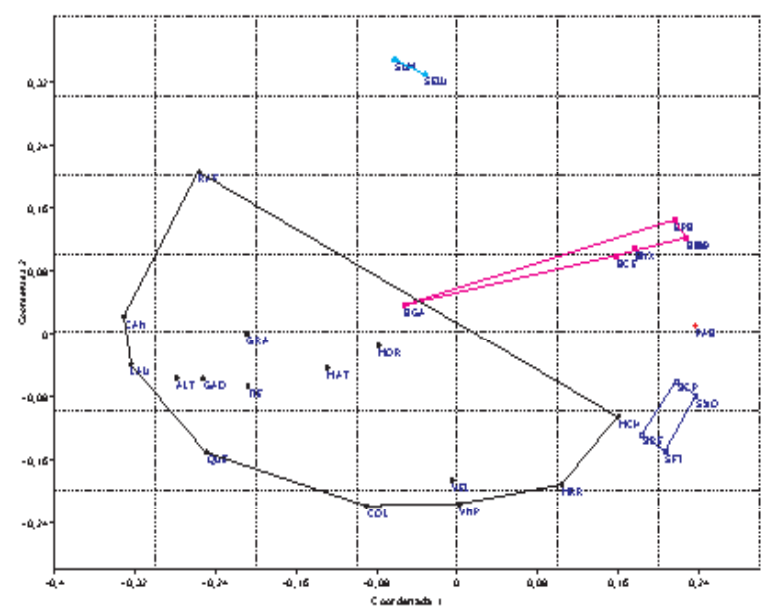

Figura 3. Análisis de coordenadas utilizando la matriz de 9 variables cualitativas. Proyección de las OTUs en el espacio de las coordenadas 1 y 2 . Abreviaturas en el texto.

está dentro de la nube de los buchones. Es el carácter referido a la posición de las alas en reposo el que define mejor el grupo de vuelo funcional o acrobático (alas por encima de la cola, en reposo); el de la longitud relativa de la cola el que define mejor el grupo baleárico (cola de longitud media a larga); el del desarrollo de las carúnculas oculares el que define mejor el grupo de morfología (desarrollo notable de la carúnculas oculares); el grupo de buchones, finalmente, viene especialmente definido por los detalles de la longitud del cuello, el colgado del buche y el desarrollo de las carúnculas nasales.

\section{DISCUSIÓN}

En el plano 1-2 a partir únicamente de 9 variables -grosor y longitud del pico, desarrollo de las carúnculas nasales y oculares, longitud del cuello, tamaño y colgado de buche, longitud relativa de la cola y posición de las alas en reposo respecto de la cola-, los cuatro grupos de OTUs aparecen diferenciados con claridad, correspondiendo a los grupos establecidos a priori, con un único solapamiento para el "Gavatxut", raza que, aunque Balear, tiene una clara aptitud como buchón.

A partir de esos nueve caracteres, pues, es posible diferenciar: el grupo 1, de buchones (Alteño, Canario, Colillano, Gaditano, Granadino, Jiennense, Laudino Sevillano, Marteño, Marchenero, Moroncelo, Morrillero Alicantino, Quebrado Murciano, Rafeño, Veleño y Nuevo Buchón Valenciano), caracterizados por una funcionalidad muy especial; el grupo 2, de palomas de vuelo funcional o acrobático ("Nas de Xot", "Coll Pelat", Figureta, Refilador, "Vol Català" y Chorrera); el grupo 3, razas baleáricas (Borino, "Colom d'Escampadissa", Mallorquina, "Pinta Balear") de trabajo y producción (excluyendo el "Gavatxut", raza balear que entra dentro del grupo de buchones, al que pertenece funcionalmente); y un grupo 4, puramente de morfología ("Colom d'UlI", "Flamenquilla" y "Ull de Maduixa"). Dentro del grupo de los buchones, el Rafeño se muestra como la raza más distante del conjunto.

Igualmente se destaca que el "Nas de Xot", aunque actualmente propio de la isla de Mallorca (Baleares), proviene de un grupo de antiguas variedades de palomas mensajeras que se volaban en la región levantina española (4). El "Coll Pelat", a pesar de su conspicua garlanda -carácter único en las razas estudiadas en este trabajo-, no se aparta del grupo de vuelo.

No existe una guía clara que determine qué nivel de semejanza (o de diferencia) es suficiente para establecer los diferentes niveles en una clasificación (1), por lo que la agrupación basada en los 9 caracteres propuestos en este artículo 
evita considerable subjetividad. Debe entenderse, por otro lado, que los caracteres estudiados procedían mayoritariamente de los estándares raciales; y el estándar viene a describir el prototipo -el patrón de perfección racial-; el estándar, pues, en algunos casos, podría diferir considerablemente de la descripción real de la raza -el morfotipo-, por lo que basar una clasificación en unos pocos caracteres normalmente siempre reflejados en los estándares resulta mucho más aplicativo que el uso de un gran número de variables, algunas, además, fuertemente correlacionadas.
En conclusión, partiendo únicamente de 9 características morfológicas cualitativas informativas -grosor y longitud del pico, desarrollo de las carúnculas nasales y oculares, longitud del cuello, tamaño y colgado de buche, longitud relativa de la cola y posición de las alas en reposo respecto de la cola-, las dos primeras dimensiones del análisis de correspondencia permiten una buena separación en cuatro grandes grupos raciales de las 29 razas estudiadas, según su aptitud, u origen, en el caso de las baleáricas.

\section{REFERENCIAS}

1. López EJ, Pérez G. Métodos de análisis en la Reconstrucción Filogenética. Bol. S.E.A. 1999; 26: 45-56.

2. Levy WM. Encyclopedia of Pigeon Breeds. South Carolina: Sumter SC. Sumter: Levi Publishing Co; 1965.

3. Mackrott H. Palomas de Raza. Barcelona: Omega; 1997.

4. Schille HJ. Guía de las Palomas de Raza. Cataluña, Valls: Arte Avícola; 2005.
5. Gower. A general coefficient of similarity and some of its properties. Biometrics $1971 ; 27(4): 857-871$.

6. Hammer $\varnothing$, Harper DAT, Ryan PD. PAST. Paleontological Statistics Software Package for Education and Data Analysis v. 1.94b. Palaeontologia Electrónica [en línea] 2001; 4(1). [acceso 21 de diciembre de 2009]; URL disponible en: http://palaeo-electronica.org/2001_1/ past/issue1_01.html. 